Proceedings

\title{
High Bandwidth Sensor Module for Mobile Robot Applications-Wind Tunnel Characterization ${ }^{+}$
}

\author{
Timothy A. Vincent, Yuxin Xing, Marina Cole * and Julian W. Gardner \\ School of Engineering, University of Warwick, Coventry, UK; T.A.Vincent@warwick.ac.uk (T.A.V.); \\ Y.Xing@warwick.ac.uk (Y.X.); J.W.Gardner@warwick.ac.uk (J.W.G.) \\ * Correspondence: marina.cole@warwick.ac.uk; Tel.: +44-24765-23546 \\ + Presented at the Eurosensors 2017 Conference, Paris, France, 3-6 September 2017.
}

Published: 25 August 2017

\begin{abstract}
We have developed a sensor module containing high-bandwidth VOC and gas sensors (including novel acoustic, custom MOX and low-cost NDIR) to evaluate the concentration of gases in a hazardous environment. We report upon the initial characterization of two MOX gas sensors $(1 \mathrm{~mm} \times 1 \mathrm{~mm})$ with coatings of $\mathrm{Pd} / \mathrm{Pt}$ doped $\mathrm{SnO}_{2}$ and pure $\mathrm{WO}_{3}$ inside a wind tunnel to plumes of acetone and ethanol (<10 ppm). The orientation and position of the sensor module in the tunnel was investigated. The unit was tested in a real-world environment with a VOC (propanol); fluctuating sensor responses demonstrated the formation of gas plumes.
\end{abstract}

Keywords: gas sensing; mobile robot; VOC; metal oxide; acetone; ppm concentrations

\section{Introduction}

Mobile robots are important tools used by civil authorities to investigate disaster environments, i.e., before human rescue teams enter hazardous sites. Visually unidentifiable dangers, such as toxic gases, place firefighter personnel at risk when they enter disaster zones. Identification of the source of any leaking gases or volatile organic compounds (VOCs) in disaster environments would greatly assist rescue operations. We have developed a module with an array of gas sensors that is capable of detecting a range of hazardous VOCs and gases such as $\mathrm{CO}, \mathrm{NO}_{2}$, acetone, toluene and $\mathrm{CO}_{2}$. The module has been developed for a tracked robot which can quickly explore its surroundings for urgent rescue missions. High bandwidth sensors (fast response, $<10 \mathrm{~s}$ ) were developed for use in this module, capable of rapidly identifying gases as the robot travels. Preliminary results from the module on a mobile robot are shown in a real-world environment and compared to a commercial PID sensor.

The sensor module was tested inside a custom-designed wind tunnel with adjustable wind speed. The unit was exposed to ethanol and acetone concentrations in the range of 2.6 to $6.8 \mathrm{ppm}$, injected through a port in the roof of the tunnel. To test the ability of the sensor module to locate the direction from which the gas source was propagating, the sensor unit was rotated inside the wind tunnel. Benchmark response levels were measured when the module was facing towards the injection port. The distance from the injection source to the module was also varied. In its current form, one inlet located $\sim 5 \mathrm{~cm}$ above the tunnel floor was used to sample the gas. A second switchable inlet, located $\sim 20 \mathrm{~cm}$ above the floor, is considered to sample gases which are less dense than air (e.g., CO).

\section{Background}

Locating survivors in collapsed buildings after an earthquake is a common application for rescue robots fitted with thermal imaging cameras and $\mathrm{CO}_{2}$ gas sensors [1]. These robots are able to inspect visual damage, but do not provide detailed information regarding hazards, which are not visible on camera images, for human entry (i.e., hidden leaking gas sources). Underground car parks are noted 
as particularly hazardous for firefighters, where visibility is poor and the source of the fire can be hard to identify [2]. Industrial explosions are another example where VOCs can be involved.

It is proposed gas sensors on a mobile robot could be used to help fire fighters identify hazardous items (e.g., leaking gas cylinders or pipes). The robot could also be used to provide information to guide a rescue operation. To facilitate use in disaster environments, the robot and the sensor unit are designed to withstand high temperatures (e.g., if a building is on fire). A fire can cause the temperature to reach between $200{ }^{\circ} \mathrm{C}$ and $300{ }^{\circ} \mathrm{C}$ at ground level [3].

In this work a wind tunnel was developed to simulate the plumes of gas that can be found in the application environments of a mobile exploration robot. The purpose of the custom wind tunnel was to trial the high bandwidth sensors with low ppm concentrations of VOCs with short exposure times. Previous reports have demonstrated the response of commercial metal oxide (MOX) sensors to plumes of gases in wind tunnels, but have been limited by long injection periods (100 s) and slow sensor response times ( $20 \mathrm{~s})[4,5]$. In our tests, the gas sensors were exposed to short pulses of VOCs (less than $5 \mathrm{~s}$ ), thus allowing a fast response and recovery of the gas sensors to be assessed.

The custom wind tunnel was designed to allow adjustable wind speeds from 1.8 to $2.3 \mathrm{~m} / \mathrm{s}$. Plumes of gas are generated along the length of the wind tunnel $(0.8 \mathrm{~m})$, which was designed to be half-cylinder shaped ( $0.5 \mathrm{~m}$ width, $0.25 \mathrm{~m}$ height) to prevent the sensor module disrupting the mixing of gases inside the tunnel. Ethanol and acetone VOCs were injected into the tunnel in liquid spray form through a port in the top with concentrations ranging from 0.9 to $6.8 \mathrm{ppm}$.

The module, shown in Figure 1a, is designed to perform gas analysis during rescue operations, so it is incorporated with a robot in real world applications. Figure $1 \mathrm{~b}$ shows the module mounted on a mobile exploration robot. The sensor unit communicates to the mobile robot via a micro USB connection. The unit requires a $5 \mathrm{~V}$ power supply ( $3 \mathrm{~W}$ maximum required). The sensor components operate at low power ( $3.3 \mathrm{~V}$ on board regulated supply), but high power components are needed, such as a pump and three valves, to control the gas flow through the module. In the case of the internal temperature of the unit rising above $70^{\circ} \mathrm{C}$, two valves are used to isolate the unit. Each valve required $0.50 \mathrm{~W}$ power to open (normally closed) and the pump requires $0.48 \mathrm{~W}$ while the unit is sampling gas. The module has two gas sampling inlets to sample gases that are of either lower or greater density than air $\left(1225 \mathrm{~kg} / \mathrm{m}^{3}\right)$. Tubes are connected to the inlets and positioned accordingly on the mobile robot; a third valve controls the switching between the two inlets.
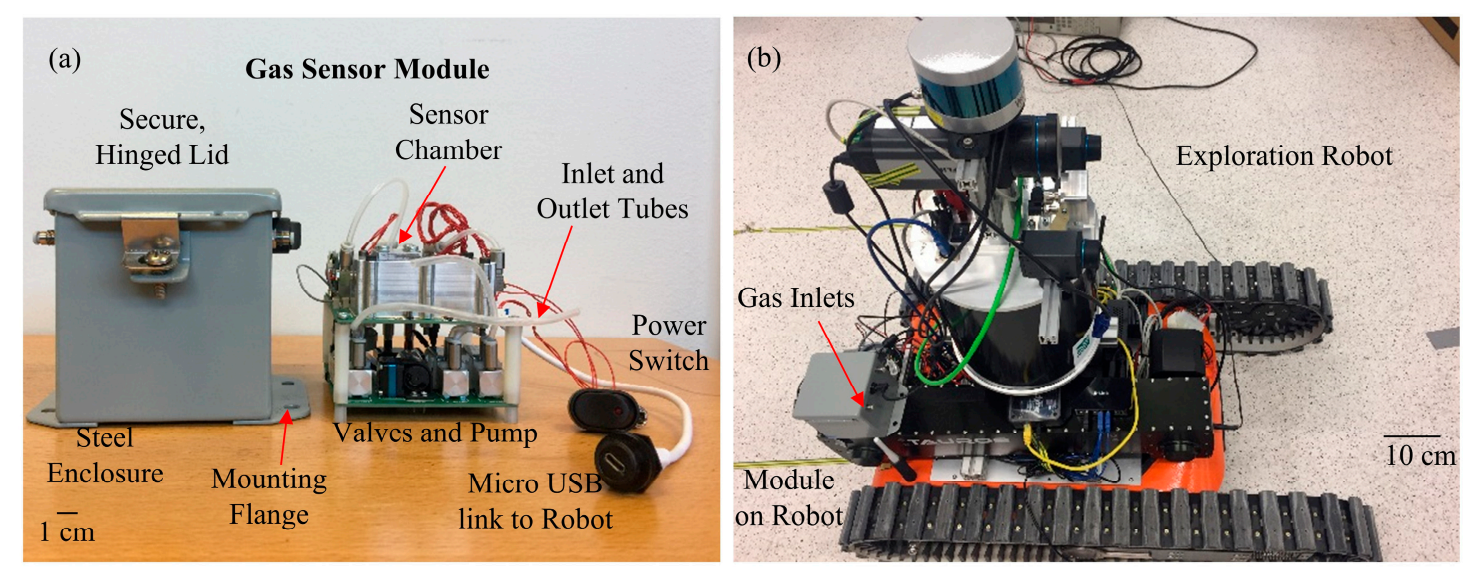

Figure 1. (a) Photograph of the second generation gas sensor module enclosure and internal components; (b) Photograph of the sensor module mounted on the rear of the tracked exploration robot. 


\section{Methodology}

Pulse width modulation (PWM) control of the inlet fan was used to adjust the wind speed inside the tunnel. The range of speeds was similar to the wind speeds generated by the movement of the mobile robot when exploring a hazardous environment. The gas inside the tunnel is sampled using a pump located inside the sensor module ( 200 SCCM). A fan (speed $2600 \mathrm{rpm}, 200 \mathrm{~mm}$ diameter) located at the tunnel inlet, generated a turbulent air flow. Preliminary smoke tests demonstrated the cylindrical tunnel allowed air to be well distributed around the cross-section area.

The concentration of the VOC injected into the tunnel was controlled by varying the number of liquid sprays through the inlet port. It was found on average $0.054 \mathrm{~g}$ of liquid was dispersed per spray. A test pattern of 3, 5, 8, 5, 3 sprays was employed with 3 min between each injection for the sensors to return to baseline. Liquid acetone and ethanol have similar density $\left(790 \mathrm{~kg} / \mathrm{m}^{3}\right.$ and 789 $\mathrm{kg} / \mathrm{m}^{3}$, respectively). Given the static area of the wind tunnel $\left(0.08 \mathrm{~m}^{2}\right)$ and assuming atmospheric pressure at $20^{\circ} \mathrm{C}$ temperature, a single spray would inject a $\sim 855 \mathrm{ppb}$ plume of either compound.

The sensor unit was positioned at the end of the wind tunnel ( $\sim .6 \mathrm{~m}$ horizontal distance from the injection port) for these experiments. The module contains the following sensors: non-dispersive infrared (NDIR, low-cost, low power device developed for $\mathrm{CO}_{2}$ sensing), solidly mounted resonator (SMR, high bandwidth device for VOC sensing), two MOX sensors (MOX1, Pd/Pt doped $\mathrm{SnO}_{2}$ and $\mathrm{MOX} 2, \mathrm{WO}_{3}$ coated device), temperature and humidity sensor (GE ChipCap2) and particle sensor (Fairchild H21A3). The MOX sensors (operated at $300^{\circ} \mathrm{C}$ ) are based on an SOI micro hotplate (AMS Sensors) [6]. Response data are recorded (5 Hz sampling rate) by a Freescale KL25Z microcontroller.

\section{Results}

Acetone was injected into the wind tunnel, photograph Figure 2a,b, with the spray pattern discussed above. Figure 2c shows a comparison between the MOX1 sensor responses when the unit (facing tunnel inlet) was located $0.6 \mathrm{~m}$ and then $0.2 \mathrm{~m}$ from the VOC port. The $\mathrm{SnO}_{2}$ coated sensor produced a larger response compared to the $\mathrm{WO}_{3}$ coated device (MOX2 data not shown; average resistance change of $1.2 \%$ compared to $3.8 \%$ for MOX1). When the module was located further from the VOC source, the response time for MOX1 increased compared to when the module was closer to the source (averages of $3.4 \mathrm{~s}$ and $2.6 \mathrm{~s}$, respectively), although the magnitude of the response increased by $2.3 \%$. The decrease in sensor magnitude conversely with distance to source demonstrates the VOC mixing with the air in the tunnel and broadening of the initially short pulses.
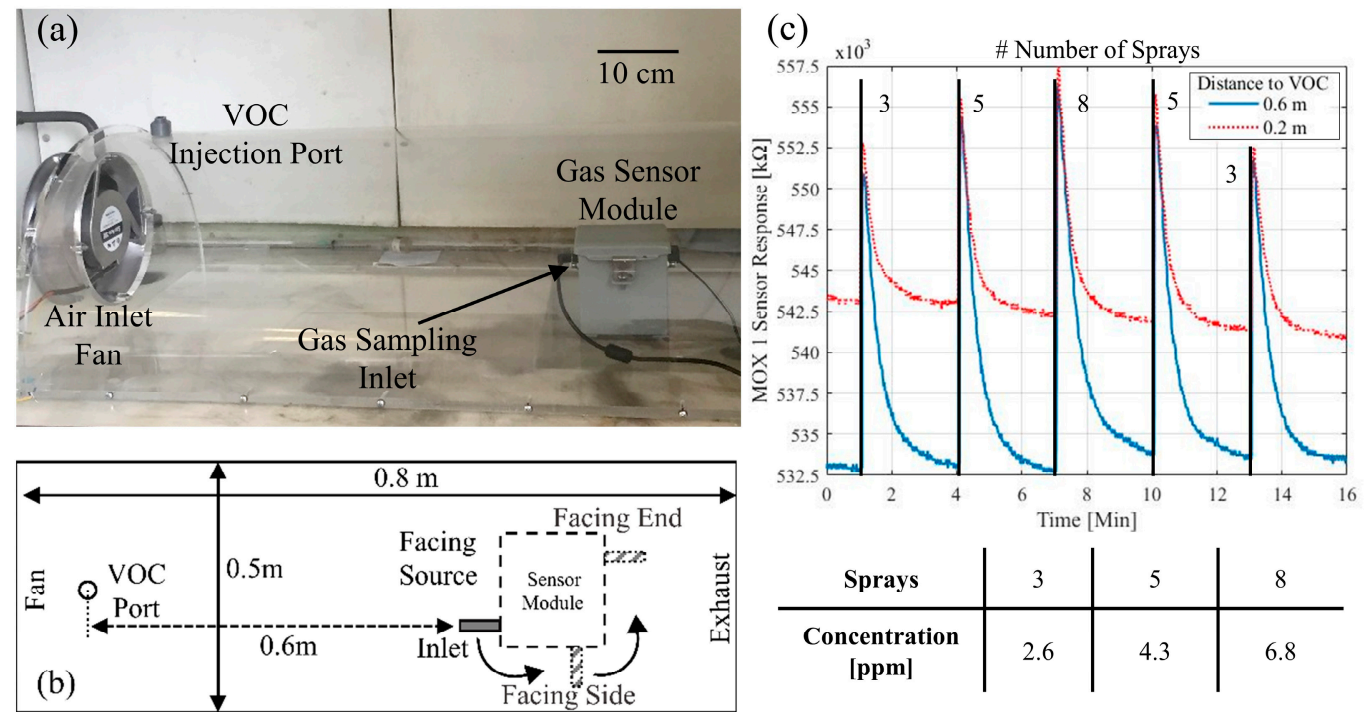

Figure 2. (a) Photograph of wind tunnel with high bandwidth sensor module (located $0.6 \mathrm{~m}$ from VOC inlet); (b) diagram to show positioning of sensor module in tunnel; (c) Response from MOX1 sensor to sprays of acetone into the wind tunnel ( $0.6 \mathrm{~m}$ and $0.2 \mathrm{~m}$ distances shown). 
The sensor module was rotated inside the tunnel to investigate if the orientation of the module sampling inlet affected the MOX sensors responses, for example if the robot turns. The orientation was varied as shown by the arrows in Figure $2 b$, so the gas inlet faced either the left side or exhaust (end) of the tunnel. Figure 3a shows the reduced response to ethanol concentrations when the sampling inlet faced the tunnel exhaust, $0.45 \%$ and $0.37 \%$ lower for MOX 1 and MOX2, respectively.

The sensor unit was compared to a commercial PID sensor to verify that plumes of gas could create a decrease in sensor output reading, regardless of the distance from the VOC source. These experiments were performed in a real world environment (temperature and humidity etc. not controlled). The response from the sensors is shown in Figure $3 \mathrm{~b}$. The fluctuating outputs shown for both the MOX and PID sensors demonstrate the formation of gas plumes in the environment (distance to source and output concentration kept constant).
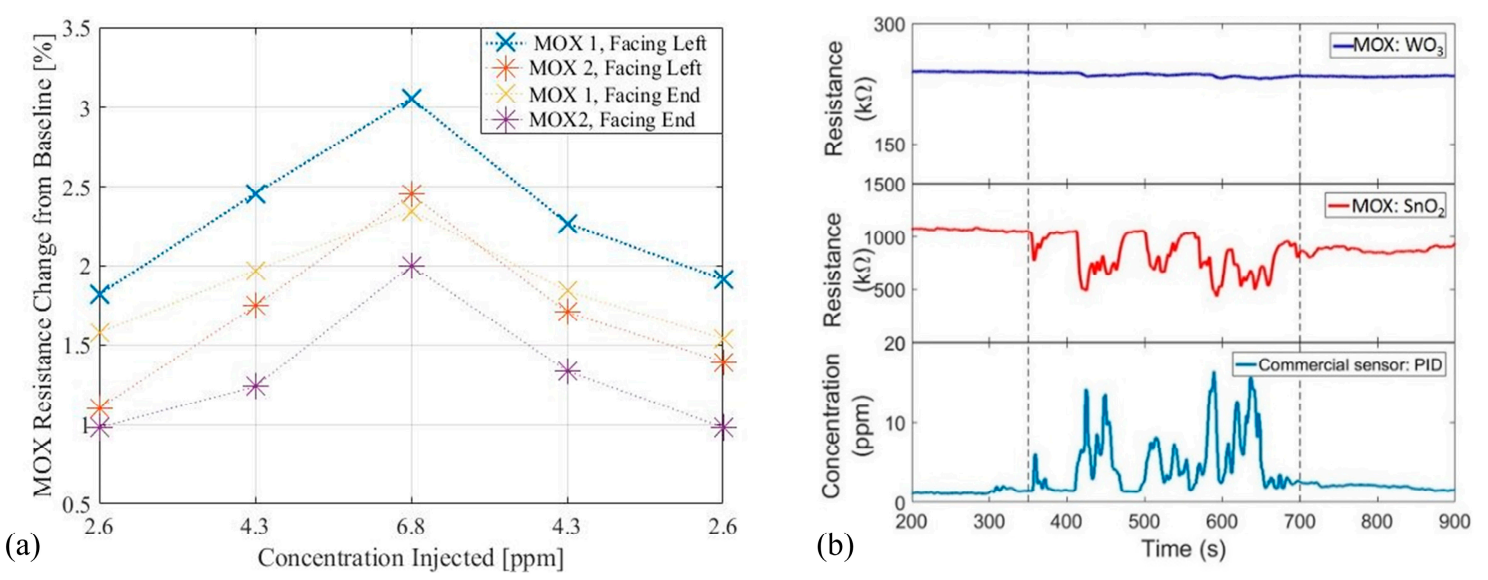

Figure 3. (a) Comparison of change in response from baseline value of MOX sensors due to injections of ethanol when unit rotated in the wind tunnel; $(\mathbf{b})$ Response of MOX sensors to plumes of propanol (0.7 $\mathrm{m}$ distance) in real world environment (vertical lines indicate start and end of injection period).

\section{Conclusions}

A sensor unit has been developed for use on a mobile robot to assess the gases present in hazardous environments. Plumes of acetone and ethanol have been produced in a wind tunnel to evaluate the detection ability of the high bandwidth sensors to low concentrations (2.6 to $6.8 \mathrm{ppm}$ ) of VOCs injected over a short period $(<5 \mathrm{~s})$. $\mathrm{A} \mathrm{SnO}_{2}$ coated sensor produced a higher response to the VOCs compared to the $\mathrm{WO}_{3}$ coated device, on average, $3.8 \%$ change in resistance compared to the $1.2 \%$ in $\mathrm{WO}_{3}$ device. The orientation of the gas sampling inlet was varied. A lower response was found when the unit faced the exhaust $(0.45 \%$ and $0.37 \%$ reduction for MOX1 and MOX2 respectively). The effect of pulse broadening has been investigated in a real world environment. Plumes of gas were observed with a commercial PID and the research MOX sensors. Further work involves developing an inverse filter to recover a step change in gas concentration from the attenuated MOX sensor response.

Acknowledgments: This work was funded in part by the H2020 ICT project 'Smokebot' (645101).

Conflicts of Interest: The authors declare no conflict of interest. The founding sponsors had no role in the design of the study; in the collection, analyses, or interpretation of data; in the writing of the manuscript, and in the decision to publish the results.

\section{References}

1. Matsuno, F.; Sato, N.; Kon, K.; Igarashi, H.; Kimura, T.; Murphy, R. Utilization of robot systems in disaster sites of the great eastern Japan earthquake. In Field and Service Robotics: Results of the 8th International Conference; Springer: Heidelberg, Germany, 2014; pp. 1-17.

2. Zhang, X.G.; Guo, Y.C.; Chan, C.K.; Lin, W.Y. Numerical simulations of fire spread and smoke movement in an underground car park. Build. Environ. 2007, 42, 3466-3475. 
3. Crewe, R.J.; Stec, A.A.; Walker, R.G.; Shaw, J.E.; Hull, T.R.; Rhodes, J.; Garcia-Sorribes, T. Experimental results of a residential house fire test on tenability: Temperature, smoke and gas analyses. J. Forensic Sci. 2014, 59, 139-154.

4. Fonollosa, J.; Rodríguez-Luján, I.; Trincavelli, M.; Huerta, R. Dataset from gas sensor array in turbulent wind tunnel. Data Brief. 2015, 3, 169-174.

5. Martinez, D.; Clotet, E.; Tresanchez, M.; Moreno, J.; Jiménez-Soto, J.M.; Magrans, R.; Marco, S.; Palacín, J. First characterization results obtained from a wind tunnel designed for indoor gas source detection. In Proceedings of the 2015 International Conference on Advanced Robotics (ICAR), Istanbul, Turkey, 27-31 July 2015; pp. 629-634.

6. Urasinska-Wojcik, B.; Vincent, T.A.; Chowdhury, M.F.; Gardner, J.W. Ultrasensitive $\mathrm{WO}_{3}$ gas sensors for $\mathrm{NO}_{2}$ detection in air and low oxygen environment. Sens. Actuators B Chem. 2017, 239, 1051-1059.

(C) 2017 by the authors. Licensee MDPI, Basel, Switzerland. This article is an open access article distributed under the terms and conditions of the Creative Commons Attribution (CC BY) license (http://creativecommons.org/licenses/by/4.0/). 